\title{
Three-dimensional elasto-plastic soil modelling and analysis of sauropod tracks
}

Eugenio Sanz, Antonio Arcos, Carlos Pascual, and Ignacio Menendez Pidal

Acta Palaeontologica Polonica 61 (2), 2016: 387-402 doi:http://dx.doi.org/10.4202/app.00098.2014

This paper reports the use of FEA (Finite Element Analysis) to model dinosaur tracks. Satisfactory reproductions of sauropod ichnites were simulated using 3D numerical models of the elasto-plastic behaviour of soils. Though the modelling was done of ichnites in situ at the Miraflores I tracksite (Soria, Spain), the methodology could be applied to other tracksites to improve their ichnological interpretation and better understand how the type and state of the trodden sediment at the moment the track is created is a fundamental determinant of the morphology of the ichnite. The results obtained explain why the initial and commonly adopted hypothesis - that soft sediments become progressively more rigid and resistant at depth-is not appropriate at this tracksite. We explain why it is essential to consider a more rigid superficial layer (caused by desiccation) overlying a softer layer that is extruded to form a displacement rim. Adult sauropods left trackways behind them. These tracks could be filled up with water due to phreatic level was close to the ground surface. The simulation provides us with a means to explain the differences between similar tracks (of different depths; with or without displacement rims) in the various stratigraphic layers of the tracksite and to explain why temporary and variable conditions of humidity lead to these differences in the tracks. The simulations also demonstrate that track depth alone is insufficient to differentiate true tracks from undertracks and that other discrimination criteria need to be taken into account. The scarcity of baby sauropod tracks is explained because they are shallow and easily eroded.

Key words: Sauropoda, ichnology, 3D, mathematical simulation, elasto-plastic model, soil mechanics, Spain, Miraflores tracksite.

Eugenio Sanz [esanz@caminos.upm.es], Antonio Arcos [antonio.arcos@caminos.upm.es], Carlos Pascual [CAPASCUAL-1@telefonica.net ], Ignacio Menendez Pidal [ignacio.menendezpidal@upm.es] (corresponding author), Escuela de Caminos, Universidad Politécnica de Madrid, Campus Ciudad Universitaria C/ Profesor Aranguren 3, 28040 Madrid, Spain. 
This is an open-access article distributed under the terms of the Creative Commons

Attribution License (for details please see creativecommons.org), which permits unrestricted use, distribution, and reproduction in any medium, provided the original author and source are credited.

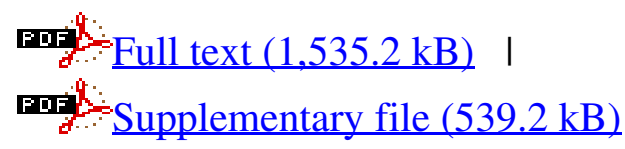

